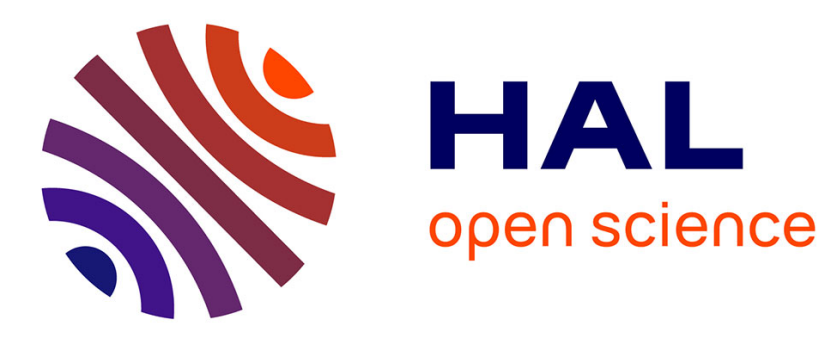

\title{
Edge dislocations in copolymer lamellar films
}

\author{
M. Turner, M. Maaloum, D. Ausserré, J.-F. Joanny, M. Kunz
}

\section{To cite this version:}

M. Turner, M. Maaloum, D. Ausserré, J.-F. Joanny, M. Kunz. Edge dislocations in copolymer lamellar films. Journal de Physique II, 1994, 4 (4), pp.689-702. 10.1051/jp2:1994156 . jpa-00247992

\section{HAL Id: jpa-00247992 https://hal.science/jpa-00247992}

Submitted on 1 Jan 1994

HAL is a multi-disciplinary open access archive for the deposit and dissemination of scientific research documents, whether they are published or not. The documents may come from teaching and research institutions in France or abroad, or from public or private research centers.
L'archive ouverte pluridisciplinaire HAL, est destinée au dépôt et à la diffusion de documents scientifiques de niveau recherche, publiés ou non, émanant des établissements d'enseignement et de recherche français ou étrangers, des laboratoires publics ou privés. 
Classification

Physics Abstracts

$61.30-68.00-61.25 \mathrm{H}$

\title{
Edge dislocations in copolymer lamellar films
}

\author{
M. S. Turner $\left({ }^{1,4}\right)$, M. Maaloum $\left({ }^{2}\right)$, D. Ausserré $\left({ }^{2}\right)$, J-F. Joanny $\left({ }^{1}\right)$ and M. Kunz $\left({ }^{3}\right)$ \\ ${ }^{1}$ Institut Charles Sadron, 6 rue Boussingault, 67083 Strasbourg Cedex, France \\ $\left({ }^{2}\right)$ Université du Maine, URA 807, B.P. 535, 72017 Le Mans Cedex, France \\ ( ${ }^{3}$ ) Ciba-Geigy A.G., Basel CH4002, Switzerland \\ $\left({ }^{4}\right)$ Cavendish Laboratory, Madingley Road, Cambridge CB3 0HE, U.K.
}

(Received 6 August 1993, revised 5 January 1994, accepted 6 January 1994)

\begin{abstract}
Thin films of symmetric diblock copolymers on a flat solid surface form lamellae parallel to the substrate. The uppermost layer of the film may be incomplete and made of domains with a thickness equal to the lamellar period $L$. We investigate these domains both theoretically and experimentally. The domain edge is associated with a dislocation in the lamellar order which has been observed by transmission electron microscopy. We extend the linear elasticity theory of smectic liquid crystals, to include the effect of the surface tension at the air-polymer interface. We calculate the surface profile above a domain edge and the position (depth) of the dislocation. This profile is studied by atomic force microscopy for polystyrenepolybutylmethacrylate films. The comparison between theoretical and experimental profiles allows an estimation of the smectic elastic constants and of the internal surface tension between the two blocks of the copolymer.
\end{abstract}

\section{Introduction.}

Symmetric A-B diblock copolymers self-organize in the melt at low temperature and form mesophases with a lamellar symmetry [1]. Thin lamellar copolymer films can be cast onto a solid substrate by spin coating [2-6]. Both the solid surface and the free surface of the film select the copolymer block that lowers the interfacial tension there and the lamellar layers lie parallel to the substrate. For the polystyrene-polybutylmethacrylate (PS/PBMA) copolymer films considered in section 3 below, it is the polybutylmethacrylate (PBMA) block which resides at both the substrate and air interfaces. In this case, a perfect, defect-free, flat film can only be made if the film thickness is an integer multiple of the lamellar repeat period $L$. ( $L$ is the thickness of a A-BB-A sequence and is twice the thickness $h$ of a single A-B layer). When the thickness is not equal to one of these discrete values, the film locally adopts one of the "allowed" values everywhere. Due to overall volume conservation, the upper layer is incomplete and has been described in terms of "islands" or "holes" depending on whether the incomplete layer 
percolates or not [7]. Islands, for example, are finite domains on the surface which correspond to an additional A-BB-A or BA-AB lamella of finite area with a thickness $L=2 h$ inserted inside the film. In order to maintain the same polymer in contact with the air, this additional lamellar domain must be inserted below the upper layer and gives rise to an edge dislocation line. The aim of this paper is to study both theoretically and experimentally the behaviour of the domains and the distortion to the smectic ordering induced by the associated dislocations. In particular we consider in detail the important role of the surface tension which acts at the copolymer-air interface.

The elastic distortion induced by the presence of an edge dislocation in an infinite Smectic-A liquid cristal was first studied by de Gennes $[8,9]$. The local displacement of the smectic layers is described by a continuous variable $u$ which varies smoothly throughout the sample. This description neglects the discrete nature of the layered phase. One finds, using linear elasticity theory, that if a dislocation line exists along the $y$ axis, with the smectic ordering parallel to the $x-y$ plane at infinity, the distortion induced by the dislocation is essentially localized inside two parabolic regions $x^{2}= \pm 4 \kappa z$ where $z$ is along the layer normal. The penetration depth of the smectic is defined as $\kappa=\sqrt{K / B}$ where $K$ is the splay constant of the smectic and $B$ its compressional modulus.

The case of an edge dislocation in a semi-infinite smectic-A phase has been considered by Kleman [10] and Pershan [11] using the approach of image dislocations. Kleman has shown that the defect is repelled by a solid surface and attracted by a free surface. The shape of a free surface above a dislocation located at a finite depth has been calculated by Pershan who neglected the surface tension acting at the surface. The dislocation is attracted towards the free surface and resides there, at equilibrium. The effect of the surface tension $\gamma$ has been considered by Lejcek and Oswald [12] who showed that the equilibrium position of the dislocation is governed by the dimensionless surface tension $\Gamma=\gamma /(K B)^{1 / 2}$ Whenever $\Gamma<1$, the dislocation is located at the solid surface; if $\Gamma>1$, it is driven into the sample interior.

In the first part of this paper, we investigate theoretically the influence of a dislocation on the surface profile of a smectic film of finite thickness using continuum linear elasticity theory. Two cases are considered. We first study a freely suspended film where both surfaces are free to deform and have finite interfacial tensions. The smectic film deposited on a solid substrate is then considered as a limiting case where one of the interfacial tensions is infinite. We calculate the equilibrium position of the dislocation and the shape of the free surface.

The second part of the paper reports an experimental study of the free surface of lamellar copolymer films deposited on a solid substrate. The shape of the domain edges is studied by atomic force microscopy. The existence of an edge dislocation at a domain edge is confirmed by transmission electron microscopy. A quantitative comparison between the continuum theory and these experimental results is made, with good agreement even for very thin films containing only a few lamellar layers. The theory gives quantitative information regarding the position (depth) of the dislocation and the elastic constants of the lamellar phase.

\section{Theory.}

2.1 Freely SUSPENDED SMECTIC FILM. - In this section we study theoretically the equilibrium properties of a smectic-A liquid crystal film, such as the lamellar phase of diblock copolymers. We adopt a coordinate system such that, in the absence of any perturbation to the smectic order, the lamellar normal vector is parallel to the $z$-axis. We describe the local vertical displacement of a lamellar layer from its equilibrium position with the variable $u$ and take the continuum limit where $u$ varies smoothly throughout the sample. We restrict our attention to straight line edge dislocations parallel to the $y$-axis and so, by symmetry, we need 
only consider the $x-z$ plane. In the following, the free energies (and energy densities) are all per unit length in the $y$-direction. The lamellar film has infinite extent in the $x$ - $y$ plane. In the first instance it is assumed to be defect free with a thickness $H$. Our treatment is based on the following classical expression for the bulk smectic energy density $[9,10,13]$ :

$$
f(x, z)=\frac{B}{2}\left(\frac{\partial u}{\partial z}\right)^{2}+\frac{K}{2}\left(\frac{\partial^{2} u}{\partial x^{2}}\right)^{2}
$$

Here $K$ is the bending or splay modulus and $B$ is the compression modulus. The smectic penetration depth is defined as $\kappa=\sqrt{K / B}$. This expression includes only the leading order terms in an expansion of the energy density in derivatives of $u$ and is therefore only strictly valid provided $u$ is slowly varying. For diblock copolymer lamellar phases recent theoretical results [13] suggest $\kappa \approx h / 3$ and $\sqrt{K B} \approx \gamma_{\mathrm{AB}}$, where $\gamma_{\mathrm{AB}}$ is the surface tension which acts at the interface between the two segregated chemical blocks.

In what follows we calculate the lamellar conformations in the bulk by minimizing the total free energy $F=\int_{x=-\infty}^{\infty} \int_{z=0}^{H} f(x, z) \mathrm{d} x \mathrm{~d} z$. Later we include the effect of the surface tension(s) which act at the sample surface(s).

It is convenient to work in Fourier-space defining the Fourier transform $a(q, z)$ of $u(x, z)$ as

$$
u(x, z)=\frac{1}{\sqrt{2 \pi}} \int_{-\infty}^{\infty} \mathrm{e}^{\imath q x} a(q, z) \mathrm{d} q
$$

where the front factor $\sqrt{2 \pi}$ is chosen for convenience. The displacement $u$ is real, we must thus require $a(-q, z)=a^{*}(q, z)$. The Fourier transform $f_{q}(z)$ of the free energy density is

$$
f_{q}(z)=\frac{1}{2}\left[B \frac{\partial a(q, z)}{\partial z} \frac{\partial a(-q, z)}{\partial z}+K q^{4} a(q, z) a(-q, z)\right]
$$

The total elastic free energy $F$ (per unit length in the $y$-direction) can be written as $F=$ $\int_{-\infty}^{\infty} \mathrm{d} q \int_{0}^{H} f_{q}(z) \mathrm{d} z=\int_{-\infty}^{\infty} \mathrm{d} q F_{q}$. The minimization of this free energy is straightforward and leads to

$$
a(q, z)=\alpha(q) \mathrm{e}^{\kappa q^{2} z}+\beta(q) \mathrm{e}^{-\kappa q^{2} z}
$$

Both $\alpha$ and $\beta$ are integration constants yet to be determined.

We now wish to calculate the lamellar displacement field due to the presence of a single edge dislocation. Such a dislocation exists when a layer fails to span the entire sample. Of particular interest is the deformation induced at the film free surface, which is the contour most easily measured experimentally [7]. We proceed by first calculating the lamellar displacement field $u(x, z)$ in a sample of thickness $H$, where $u(x, 0)$ is imposed as a boundary condition. This corresponds to the problem of a defect-free smectic film on a solid substrate, which has some general roughness (with translational symmetry in the $y$-direction). This procedure is then generalized to treat a dislocation in the interior of a smectic film by decomposing the sample into two defect-free portions, one above the dislocation and one below (see Fig. 1). We determine both the lamellar displacement field $u$ throughout the sample and the equilibrium position (height) of the dislocation.

We first include the surface energy of the air-smectic interface at $z=H$ in the free energy $F_{q}$. (In the experiments described in Sect. 3 below this corresponds to the PBMA-air interface).

$$
F_{q}=\frac{1}{2}\left(\gamma q^{2} a_{H} a_{H}^{*}+\int_{0}^{H} \mathrm{~d} z\left[B \frac{\partial a}{\partial z} \frac{\partial a^{*}}{\partial z}+K q^{4} a a^{*}\right]\right)
$$




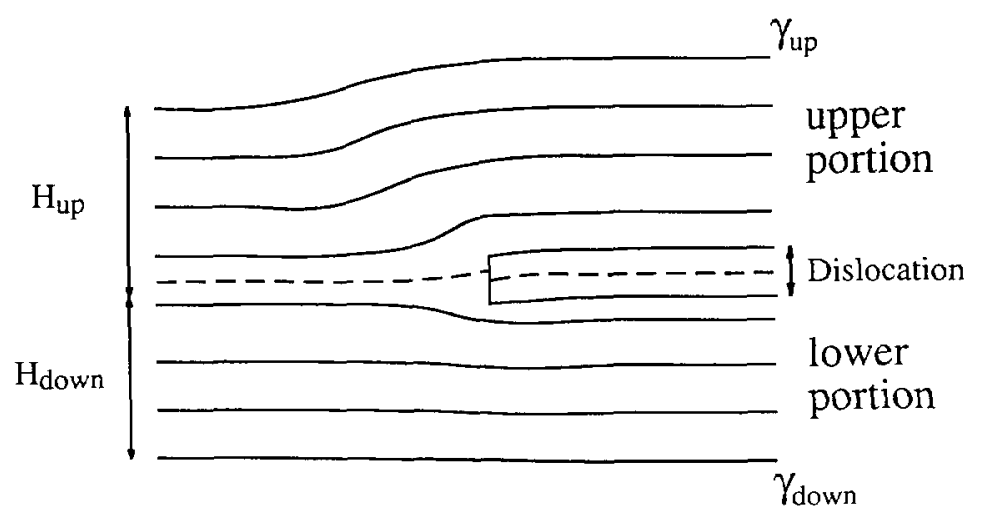

Fig. 1. - Schematic diagram showing the lamellar conformations in the vicinity of an idealized (stepfunction) edge dislocation. Note that the dislocation is flexrble.

Here $a_{H}=a(q, H)$; the new term involving the surface tension $\gamma$ is a good approximation to the interfacial energy provided that this interface has a small slope.

The displacement field above a substrate with a given roughness characterized by $a_{0}=a(q, 0)$ is given by equation (4)

$$
a(q, z)=\left(a_{H}(q)-a_{0}(q) \mathrm{e}^{-\kappa H q^{2}}\right) \frac{\sinh \kappa q^{2} z}{\sinh \kappa H q^{2}}+a_{0}(q) \mathrm{e}^{-\kappa q^{2} z}
$$

The minimization of the free energy with respect to the shape of the upper surface $a_{H}$ is equivalent to imposing the continuity of the normal stress at the free surface, it leads to:

$$
a(q, z)=a_{0}(q) \frac{\mathrm{e}^{\kappa q^{2} z}+G \mathrm{e}^{2 \kappa H q^{2}} \mathrm{e}^{-\kappa q^{2} z}}{1+G \mathrm{e}^{2 \kappa H q^{2}}}
$$

where $G=(1+\Gamma) /(1-\Gamma)$ and $\Gamma=\gamma / \sqrt{K B}$ is a dimensionless surface tension. This expression has the following boundary conditions: (i) $a=a_{0}$ when $z=0$. (ii) $a_{H}=0$ as $\gamma \rightarrow \infty$.(iii) $a_{H}=a_{0}$ when $\kappa=0$; in this limit the lamellae are incompressible and the deformation is undamped. The free energy of the smectic film above a rough solid surface reads then

$$
F_{q}=\frac{1}{2} \sqrt{K B} q^{2} a_{0} a_{0}^{*} \frac{G \mathrm{e}^{2 \kappa H q^{2}}-1}{G \mathrm{e}^{2 \kappa H q^{2}}+1}
$$

We now consider the equilibrium effect of a single edge dislocation trapped inside a bulk sample, which has a total thickness $H_{\text {tot }}$. The dimensionless surface tensions at the upper and lower bulk surfaces are $\Gamma_{\text {up }}$ and $\Gamma_{\text {down }}$ respectively. The edge dislocation is at a distance $H_{\text {down }}$ from the lower surface and a distance $H_{\text {up }}$ from the upper surface, where $H_{\text {tot }}=H_{\text {up }}+H_{\text {down }}$. The discontinuity in $u$ across the dislocation is $\Delta u(x)$, it has a Fourier transform $\Delta a(q)$. We make here no assumptions about the conformation of the dislocation, which needs not be flat.

The elastic distortion induced by the dislocation and its position at thermal equilibrium are determined by minimization of the sum of the energies of the upper and lower portions. The energy densities $F_{q}^{\text {up }}$ and $F_{q}^{\text {down }}$, for the upper and lower portions respectively, are given by (8) where $a_{0}$ is replaced by $a_{0}^{\text {up }}=\hat{a}+\Delta a / 2$ for the upper portion and $a_{0}^{\text {down }}=-\hat{a}+\Delta a / 2$ for the lower portion. Since $\hat{a}(q)$ is unknown this introduces no further assumptions about the 
conformation of the dislocation. The minimization of $F_{q}^{\text {tot }}=F_{q}^{\text {up }}+F_{q}^{\text {down }}$ with respect to $\hat{a}$ gives

$$
a_{0}^{\text {up }}=\Delta a \frac{\Lambda_{\text {down }}}{\Lambda_{\text {up }}+\Lambda_{\text {down }}} \quad a_{0}^{\text {down }}=\Delta a \frac{\Lambda_{\text {up }}}{\Lambda_{\text {up }}+\Lambda_{\text {down }}}
$$

where $\Lambda_{3}=\frac{G_{3} \mathrm{e}^{2 \kappa H_{3} q^{2}}-1}{G_{3} \mathrm{e}^{2 \kappa H_{3} q^{2}}+1}, G_{3}=\frac{1+\Gamma_{3}}{1-\Gamma_{3}}, \Gamma_{3}=\gamma_{3} / \sqrt{K B}$ and $j$ labels one of either up or down. The lamellar displacement field for general values of $H_{\text {up }}, H_{\text {down }}, \kappa, \Gamma_{\text {up }}$ and $\Gamma_{\text {down }}$ is given by equations (2), (7) and (9). The total free energy $F_{q}^{\text {tot }}$ of each $q$-mode then reads

$$
F_{q}^{\mathrm{tot}}=\frac{1}{2} \sqrt{K B} q^{2} \Delta a \Delta a^{*} \frac{\Lambda_{\mathrm{up}} \Lambda_{\mathrm{down}}}{\Lambda_{\mathrm{up}}+\Lambda_{\mathrm{down}}}
$$

In obtaining (9) and (10) we have not explicitly imposed continuity of $\partial u / \partial z$ (which is physically required so that the normal stress is continuous) since it follows naturally from minimization of the energy.

The minimization of $F^{\text {tot }}=\int F_{q}^{\text {tot }} \mathrm{d} q$ with respect to $H_{\mathrm{up}}$ (say) gives the equilibrium position of the dislocation. The free energy $F_{q}^{\text {tot }}$ given by (10) does not include any contribution from the dislocation core itself: the molecules in the core (near the edge) are forced to adopt unfavourable conformations, with an associated free energy penalty. Whilst this contribution has no effect on the equilibrium properties of interest in the present work it would provide an important contribution to the line tension acting at the dislocation edge and could be calculated from the theory of Semenov [14]. In the present theory we also have neglected the bending and compressional contributions to the free energy from the material making up the dislocation core itself. In principle it would be possible to include these contributions, at least away from the dislocation edge, at the expense of some mathematical simplicity (the conformation of the dislocation would be more intricately coupled to the free energy). However whenever the sample consists of many layers the contribution from the core bending and compression is expected to become relatively unimportant. When there are very few layers present other problems also arise: for example the validity of the continuum approximation; the linearised theory must be considered as well as whether the gradient of the sample surface is small enough for the approximation to the interfacial energy taken in (5) to be a good one. Nonetheless we expect our theory to provide useful results in many cases.

Finally, near the edge of the dislocation the displacement field $u$ is not slowly varying and the corrections to the approximate free energy (1) become important. However we retain (1) as an approximate description of the bulk free energy, an approach which is conventional in the literature. This may be justified on the grounds that we expect the contribution to the total free energy from the region near the dislocation edge to be relatively insensitive to variation of $H_{\mathrm{tot}}, \Gamma_{\mathrm{up}}$ and $\Gamma_{\mathrm{down}}$. In any case an extension of (1) to the regime where $u$ is not slowly varying is far from straightforward.

2.2 SMECTIC FILM ON A RIGID, FLAT SUBSTRATE. - Throughout section 2.2 we consider the special case of a single edge dislocation trapped in a sample of thickness $H_{\text {tot }}$, which itself resides on top of a rigid flat surface. The lower solid surface may then be modelled by taking the limit $\Gamma_{\text {down }} \rightarrow \infty$ in the results of the previous section. We make predictions for both the equilibrium depth of the dislocation below the free surface $H_{\mathrm{up}}$ and the lamellar displacement field. We give special attention to the contour of the upper (free) surface, which is the contour measured experimentally, as described in section 3 . 
2.2.1 Equilibrium dislocation position. - The equilibrium dislocation position is determined by minimizing $F^{\text {tot }}=\int F_{q}^{\text {tot }} \mathrm{d} q$. Using equation (10) we find

$$
\frac{\partial F^{\mathrm{tot}}}{\partial H_{\mathrm{up}}}=\frac{1}{2} \sqrt{K B} \int q^{2} \Delta a \Delta a^{*} \frac{\mathrm{e}^{2 \kappa H_{\mathrm{tot}} q^{2}}+G_{\mathrm{up}} \mathrm{e}^{4 \kappa H_{\mathrm{up}} q^{2}}}{\mathrm{e}^{2 \kappa H_{\mathrm{up}} q^{2}}\left(G_{\mathrm{up}} \mathrm{e}^{2 \kappa H_{\mathrm{tot}} q^{2}}+1\right)} \mathrm{d} q
$$

In the case where $\Gamma_{\text {up }}<1\left(G_{\text {up }}>0\right)$ we see by inspection of (11) that $F^{\text {tot }}$ is a monotonic increasing function of $H_{\text {up }}$. Hence in this range of $\Gamma_{\text {up }}$ the dislocation is attracted towards the free surface.

In the opposite limit $\Gamma_{\text {up }} \rightarrow \infty\left(G_{\mathrm{up}} \rightarrow-1\right)$ a similar inspection of (11) shows that $H_{\mathrm{up}} \rightarrow$ $H_{\text {tot }} / 2$ : in this limit, the dislocation is repelled from the surface, towards its equilibrium position at the center of the film. This result might be expected on symmetry grounds.

In Appendix $A$ the scaling of $H_{\text {up }}$ with $\Gamma_{\text {up }}$ is estimated in the asymptotic limit $\Gamma_{\text {up }}=1+\delta$, where $\delta \ll 1$. A sketch graph of the variation of $H_{\text {up }}$ with $\Gamma_{\text {up }}$ is shown in figure 2 .

These results are in agreement with those recently proposed by Lejcek and Oswald [12] , using the method of image dislocations.

2.2.2 Lamellar displacement field. - We now seek to determine the lamellar displacement field at the upper free surface in the presence of an edge dislocation. In what follows it is necessary to explicitly define $\Delta u(x)$, the discontinuity in $u$ due to the presence of a dislocation. For simplicity we use the following approximate form, although we expect our results to be relatively insensitive to the precise choice of $\Delta u$ near $x=0$.

$$
\Delta u(x)= \begin{cases}2 h & \text { for } x>0 \\ 0 & \text { for } x<0\end{cases}
$$

We again emphasize that the dislocation is flexible. In diblock lamellar systems $h$ corresponds to the thickness of a single A-B layer and a dislocation consists of an A-BB-A or B-AA-B double layer with a thickness $2 h$, hence (12). According to $(2) \Delta u(x)$ has a Fourier transform given by

$$
\Delta a(q)=2 \hbar / i q
$$

where $\hbar=h /(2 \pi)^{1 / 2}$

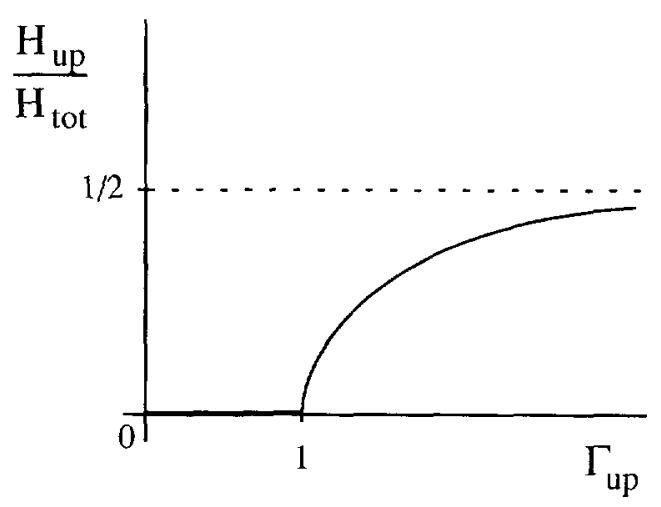

Fig. 2. - Schematic diagram showing the variation of the equilibrium depth of the dislocation $H_{\text {up }}$ (solid line), in units of the total sample thickness $H_{\text {tot }}$, with the rescaled surface tension $\Gamma_{\text {up }}$. 
Using (2), (7) and (9) we write the lamellar displacement field at the upper surface as

$$
u\left(x, H_{\mathrm{up}} ; \Gamma_{\mathrm{up}}\right)=\frac{h}{\pi} \int_{0}^{\infty} \frac{\sin q x}{q} \frac{\left(\mathrm{e}^{2 \kappa H_{\mathrm{down}} q^{2}}+1\right)\left(1+G_{\mathrm{up}}\right) \mathrm{e}^{\kappa H_{\mathrm{up}} q^{2}}}{G_{\mathrm{up}} \mathrm{e}^{2 \kappa H_{\mathrm{tot}} q^{2}}+1} \mathrm{~d} q .
$$

For the special case $\Gamma_{\text {up }}=1\left(G_{u p} \rightarrow \infty\right)$, the profile can be calculated explicitly [15].

$$
u\left(x, H_{\mathrm{up}} ; 1\right)=\frac{h}{2}\left(\operatorname{erf} \frac{x}{2 \sqrt{\kappa H_{\mathrm{up}}}}+\operatorname{erf} \frac{x}{2 \sqrt{\kappa\left(2 H_{\mathrm{tot}}-H_{\mathrm{up}}\right)}}\right) .
$$

The sharp step in $u$ at the dislocation is thus "smoothed out" by those layers between the dislocation and the surface. The upper contour is significantly distorted near $x=0$ over some length $l$, that we call the "healing length". Thus the upper lamellar layer is approximately flat for $|x|>l / 2$. For simplicity, we may define quantitatively the healing length $\lambda$ by,

$$
\lambda=2 h /\left.\frac{\partial u(x, H)}{\partial x}\right|_{x=0}
$$

In the special case $\Gamma_{\text {up }}=1$, we obtain

$$
\lambda=4 \sqrt{\kappa \pi}\left(\frac{1}{\sqrt{H_{\mathrm{up}}}}+\frac{1}{\sqrt{2 H_{\mathrm{tot}}-H_{\mathrm{up}}}}\right)^{-1}
$$

Whilst the exact solution for general $\Gamma_{\text {up }}$ is not easily obtained, one case of interest, which may often be relevant experimentally [7] is $\Gamma_{\text {up }} \gg 1$. For such values of $\Gamma_{\text {up }}$ it is possible to estimate the scaling of $\lambda$ given in equation (18) below, as described in Appendix B.

$$
\lambda \simeq\left(\kappa H_{\text {tot }} \Gamma_{\text {up }}\right)^{1 / 2}
$$

In deriving this result we have assumed that the dislocation is close to its equilibrium position in the center of the sample.

Figure 3 shows the dependence of the rescaled healing length $\lambda^{\prime}=\lambda /\left(\kappa H_{\text {tot }}\right)^{1 / 2}$ on the rescaled surface tension $\Gamma_{\text {up }}$.

In recent experiments on the PS/PBMA diblock copolymer lamellar phase [7] it was found that $\lambda$ is larger by a factor of order 20 than the estimate of reference [11] obtained by neglecting

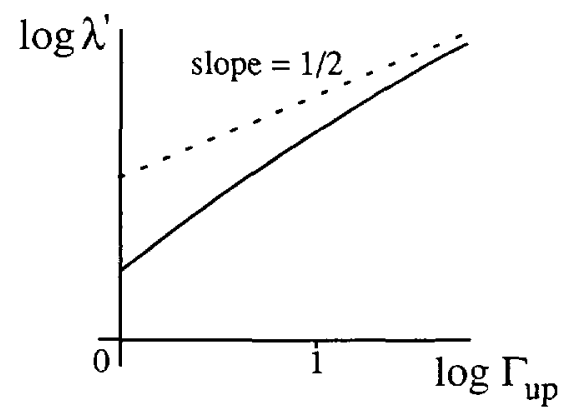

Fig. 3. - Schematic diagram showing the variation of the logarithm of the rescaled healing length $\lambda^{\prime}=\lambda\left(\kappa H_{\text {tot }} \Gamma_{\text {up }}\right)^{-1 / 2}$ (solid line) with the logarithm of the rescaled surface tension $\Gamma_{\text {up }}$. The dotted line is the asymptotic tangent with slope $1 / 2$. 
the surface tension. This difference was tentatively attributed to the effect of the air-polymer surface tension at the upper free surface of the film. As described in section 3 below the present work demonstrates that surface tension must be included and that it accounts for this apparent discrepancy.

The total elastic energy due to the dislocation $F^{\text {tot }}$ can also be calculated in the specific case where $\Gamma_{\text {up }}=1$

$$
F^{\mathrm{tot}}=\hbar^{2} \sqrt{K B} \int\left(\mathrm{e}^{-2 \kappa H_{\mathrm{down}} q^{2}}+1\right) \mathrm{d} q .
$$

It is important to recall that the contribution to the bulk smectic energy from modes which are not slowly varying is known to be inaccurately modelled by the linear elasticity theory of equation (1). Near the core leading edge this corresponds to modes $q \gtrsim h^{-1}$ We therefore introduce a cutoff in (19) and evaluate the integral only over $|q|<q_{c}$ where $q_{c} \simeq h^{-1}$ The integral diverges without this cutoff, indicating that the energy of the high $|q|$-modes are strongly overestimated. The free energy calculated this way therefore does not include the contribution of the core of the dislocation. We obtain

$$
F^{\text {tot }}=\left\{\begin{array}{ll}
2 \hbar^{2} \sqrt{K B} q_{\mathrm{c}} & \text { for } H_{\text {down }} \gg\left(2 \kappa q_{\mathrm{c}}^{2}\right)^{-1} \\
4 \hbar^{2} \sqrt{K B} q_{\mathrm{c}} & \text { for } H_{\text {down }} \ll\left(2 \kappa q_{\mathrm{c}}^{2}\right)^{-1}
\end{array} .\right.
$$

The first of these two regimes $\left(H_{\text {down }} \gg\left(2 \kappa q_{\mathrm{c}}^{2}\right)^{-1}\right)$ corresponds to copolymer lamellar samples with many layers (since for such systems [13] $\kappa \approx h / 3$ ). Neglecting all numerical constants of order unity, we may approximate $(20)$ by $F^{\text {tot }} \simeq h \sqrt{K B}$, which, for copolymer samples, gives $F^{\text {tot }} \simeq h \gamma_{\mathrm{AB}}$ (since $\sqrt{K B} \approx \gamma_{\mathrm{AB}}$ in this case [13]).

Using a method similar to that described in Appendix B, including the cutoff at $|q|=q_{\mathrm{c}}$, it is possible to estimate the scaling of $F^{\text {tot }}$ for $\Gamma_{\text {up }} \gg 1$ :

$$
F^{\mathrm{tot}} \simeq 2 \hbar^{2} \sqrt{K B} q_{\mathrm{c}}+h^{2}\left(\frac{K B \Gamma_{\mathrm{up}}}{\kappa H_{\mathrm{tot}}}\right)^{1 / 2}
$$

In deriving this result we have, for simplicity, assumed $H_{\text {up }}=H_{\text {down }}=H_{\text {tot }} / 2$, i.e. that the dislocation resides at its equilibrium position in the center of the sample. For copolymer lamellar systems with $n$ layers $\left(H_{\text {tot }}=n h\right.$ ) equation (21) reduces to the approximate result

$$
F^{\text {tot }} \simeq h \gamma_{\mathrm{AB}}+h n^{-1 / 2} \sqrt{\gamma_{\mathrm{AB}} \gamma_{\mathrm{up}}}
$$

where $\gamma_{u p}$ is the surface tension acting at the upper film surface.

\section{Experimental.}

The copolymer used in the experimental study was a polystyrene-polybutylmethacrylate (PS/PBMA) of molecular mass 82,000, with a polydispersity index of 1.04 [16]. It was synthetized using anionic polymerization and purified by liquid chromatography. The PS sequence has a mass of 43,000 and the copolymer is therefore roughly symmetric. As a consequence the microdomains appearing in the ordered state are lamellar and form a bilayered smectic arrangement characterized by a translational period $L=2 h$ of about $315 \AA$. For PS/PBMA it is the PBMA block which resides at both the silicon and air interfaces. Thin copolymer films were prepared by spin-coating a dilute solution onto freshly cleaned silicon wafers. The rotating rate was tuned in order to obtain four different film thicknesses $d_{j}$ intermediate between $j L$ and $(j+1) L$, with $j=1,2,4,5$. 
The films, initially solid and disordered were annealed under vacuum for 24 hours at a temperature of $150^{\circ} \mathrm{C}$, which is well above the glass transition temperature of both species and much below the order-disorder temperature of the pure copolymer. Smectic ordering appeared, with layers parallel to the substrate and local quantification of the film thickness to either $j L$ or $(j+1) L$ everywhere. Islands of thickness $L$ appear on a film of thickness $j L$ when an average thickness only slightly larger than $j L$ is imposed.

The presence of a dislocation loop below each island edge has been inferred previously [7]. The present experiment therefore gives a direct test of the theoretical predictions of section 2 , provided that the domains are large enough that any curvature in the dislocation loop is small and that the treatment of section 2 , based on a straight line edge dislocation, is appropriate. It has been checked experimentally that the edge profiles are insensitive to the domain radius for the size of domains investigated here. The Atomic Force Microscope (AFM) study of the free surface of the films was carried out after cooling the samples to room temperature, which is below the glass transition temperature for either blocks, leaving the samples in a solid state (for PS $T_{\mathrm{g}}=100^{\circ} \mathrm{C}$, for PBMA $\left.T_{\mathrm{g}}=30^{\circ} \mathrm{C}[17]\right)$. Because of the very high viscosity of the layered structure the smectic ordering and its defects are supposed to be vitrified during the quench, without appreciable modification. Once in the solid state the free surface topography can be studied quantitatively using the AFM in the contact mode. A Nanoscope II instrument was used (Digital, California). Previous similar measurements on the same system had shown that the thickness profile above a domain edge was extremely flat, with a maximum slope $\frac{\mathrm{d} u}{\mathrm{~d} x} \approx 0.1$. This guarantees the absence of profile distortion due to a convolution with the probe shape (AFM tip). The use of different contact forces demonstrated the absence of profile distortion due to sample tip interactions. Finally a controlled amount of homopolymer was added in order to check that residual homopolymer is not responsible for the profile flattening [16] which is attributed here to the effect of the air-polymer surface tension.

We mention at this point some possible sources of error: the dynamics of the dislocation perpendicular to the smectic ordering are expected to be very slow and one should bear in mind the possibility that its position may be "frozen in" during sample preparation, not necessarily at the equilibrium depth. However the experimental results agree well with the equilibrium theory, this seems to indicate that the annealing process is sufficiently thorough to leave the system at equilibrium. One other possible source of error regards the smoothness of the substrate. Inhomogeneities on the surface of the substrate could have dramatic effects, strongly pinning the dislocation, not necessarily at the expected depth below the free surface. In the present work silicon wafers were used which have a roughness much lower than $50 \AA$.

The AFM profiles presented in figures 4-7 are obtained by measuring the surface profile of the quenched copolymer sample at each of the four film thicknesses. The measurements are made normally to the domain edges. The full lines on each figure represent the best numerical evaluation of the Fourier integral in equation (14) after a three-parameter fit based on quadratic error minimization. The three fitted parameters are the characteristic length $\kappa$, the dimensionless surface tension $\Gamma_{\text {up }}$ and the dislocation depth $H_{\text {up }}$. Their values are given in table I for each of the four values of $H_{\text {tot }}=j L$. The overall step height $L$ may be extracted from figures 4-7 and is found to have a constant value of $315 \AA$. The parameters $\kappa$ and $\Gamma_{\text {up }}$ are found to remain constant, to within $1 \mathrm{~nm}$ and 1 unit respectively. This gives some confidence in the fitting procedure since these are material parameters which are not expected to vary with the film thickness. Combining these parameters, and using the fact that PBMA is present at the free surface with a surface tension $\gamma_{\text {up }}=23.5 \mathrm{dynes} / \mathrm{cm}$ in the melt [18], we can estimate the elastic constants for the lamellar sample. We find $B=10^{6}$ dynes $/ \mathrm{cm}^{2}$ and $K=10^{-6}$ dynes. These values are comparable to those found in classical bilayered smectics 


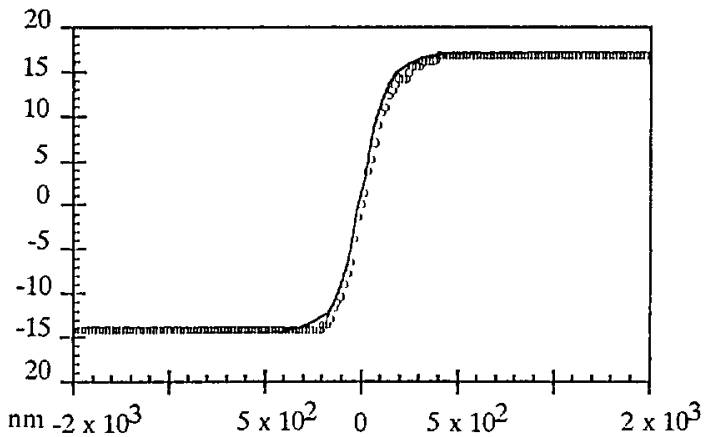

Fig. 4

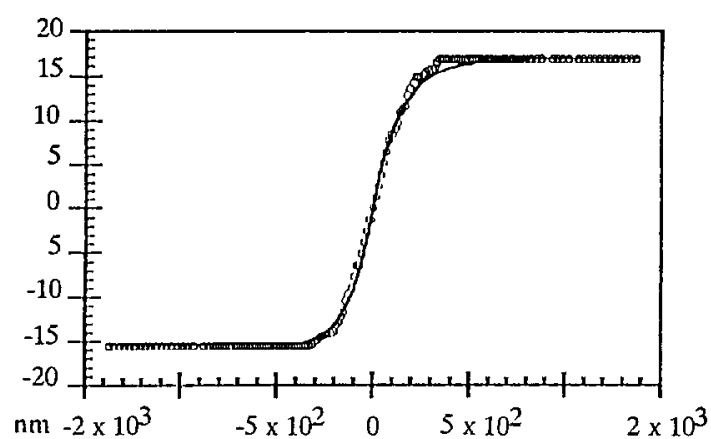

Fig. 5

Fig. 4. - A plot of the local film thickness against position, measured along an axis parallel to the substrate and normal to the domain edge. The average thickness $d_{1}$ is intermediate between $L$ and $2 L$. Dots display experimental results. The full line was obtained numerically from equation (19) for the best parameters $H_{\mathrm{up}}, \Gamma_{\mathrm{up}}$ and $\kappa$.

Fig. 5. - Same as figure 4 for an average film thickness $d_{2}$ such that $2 L<d_{2}<3 L$.

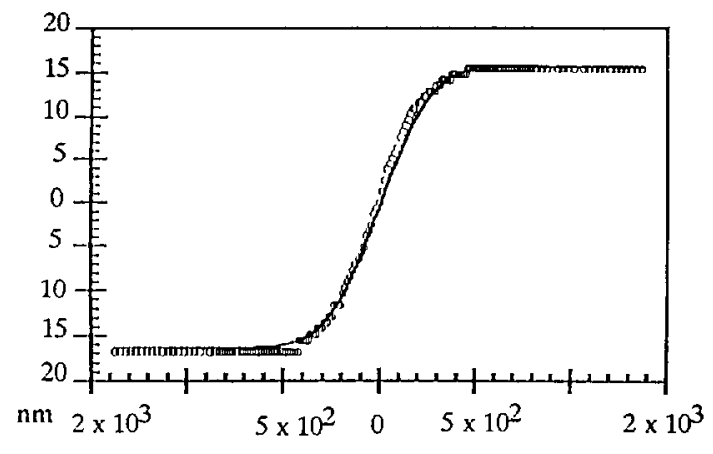

Fig. 6

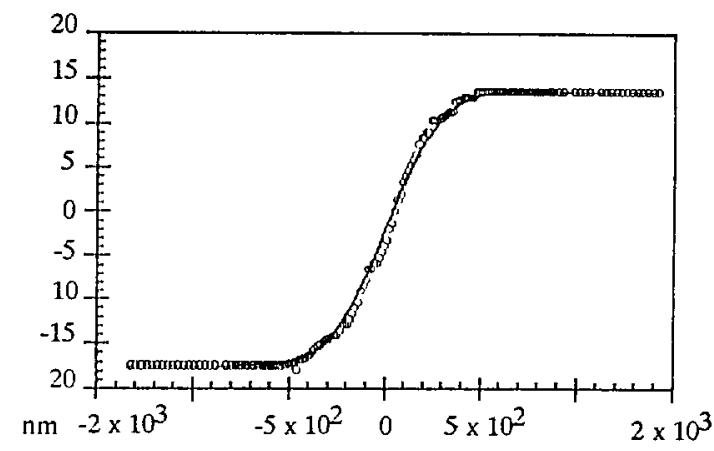

Fig. 7

Fig. 6. - Same as figure 4 for an average film thickness $d_{4}$ such that $4 L<d_{4}<5 L$.

Fig. 7. - Same as figure 4 for an average film thickness $d_{5}$ such that $5 L<d_{5}<6 L$.

[12]. Using the relations $K \approx \gamma_{\mathrm{AB}} h / 3$ and $B \approx 3 \gamma_{\mathrm{AB}} / h$ [13] we can estimate the value of $\gamma_{\mathrm{AB}}$, which is a quantity that is difficult to access directly. We find

$$
\gamma_{\mathrm{AB}}=\sqrt{K B}=0.9 \text { dynes } / \mathrm{cm}
$$

which is a quite reasonable value. Also $h$ can be estimated as $300 \AA$. Although this has the right order of magnitude, it is not in agreement with the value of $L=2 h$. The discrepancy may be due to the inadequacy of the molecular model of reference [13] for the copolymer which assumes that all the chain ends reside on the lamellar surfaces or to the finite molecular weight in the experiment. This would also affect the value of the internal surface tension $\gamma_{A B}$.

It is interesting to compare the free energy of the dislocation in the film to the free energy of the same lamellar domain deposited on the surface of a dislocation free film. In this last case 
Table I. - Parameters obtained from the fit of the surface contour to the present theory for copolymer lamellar films of various thicknesses.

\begin{tabular}{||c|c|c|c|c||}
\hline$j$ & 1 & 2 & 4 & 5 \\
\hline$H_{\text {tot }} \AA$ & 315 & 630 & 1260 & 1575 \\
\hline$\kappa \AA$ & 100 & 104 & 102 & 106 \\
\hline$\tau_{\text {up }}$ & 27 & 27 & 27 & 26 \\
\hline$H_{\text {up }} \AA$ & 10 & 20 & 50 & 350 \\
\hline \hline
\end{tabular}

the polymer with the higher surface tension is in contact with the air at the edge of the domain. The corresponding cost in interfacial energy is $F_{\mathrm{d}}=\Delta \gamma h$ where $\Delta \gamma$ is the difference between the surface tensions of the blocks of the copolymer, of order 10 dynes/cm for PS-PBMA. The free energy of the dislocation is given by (22) to which one must add the core energy. A scaling analysis suggests that the core energy is of order $F_{\mathrm{c}} \simeq h \gamma_{\mathrm{AB}}$. The very small value of $\gamma_{\mathrm{AB}}$ in (23) shows that the creation of a dislocation inside the film is favorable.

The depth of the dislocation below the free surface also depends on the total film thickness. There is only a small apparent change in $H_{\mathrm{up}}$ as the sample thickness is increased to $1260 \AA$. However, on increasing the film thickness further to $1575 \AA$ there is a sharp jump to $H_{\text {up }}=350 \AA$. The amplitude of this jump clearly corresponds to the thickness of one lamellar layer. Finally figure 8 shows the variation of the profile width $\lambda$, as defined by (16), with the square root of the film thickness. A linear dependence is observed, in agreement with (18).

This work has been based on the assumption that steps in the free surface are associated with the presence of a single dislocation inside the copolymer film. Experimental verification of this is given by figure 9 which reproduces a TEM micrograph of an ordered copolymer film deposited on a hard polymeric substrate. The copolymer is slightly different from that discussed above, it is polystyrene-polymethylmethacrylate with a molecular mass of 102,000 and a polydispersity index of 1.05. Thin films exhibit the same kind of ordering and surface steps as described in

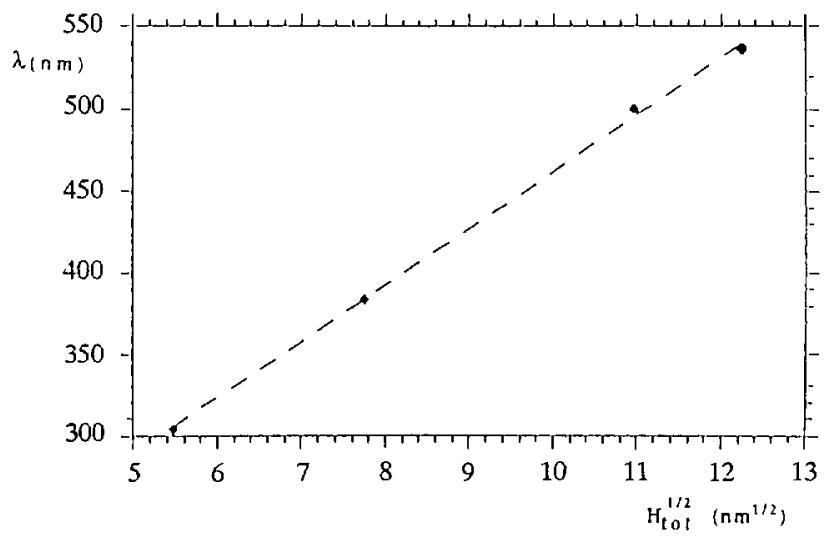

Fig. 8. - Width $\lambda$ (in $\mathrm{nm}$ ) of the thickness profile as a function of the square root of the total film thickness $H_{\text {tot }}\left(\right.$ in $\mathrm{nm}^{1 / 2}$ ) 


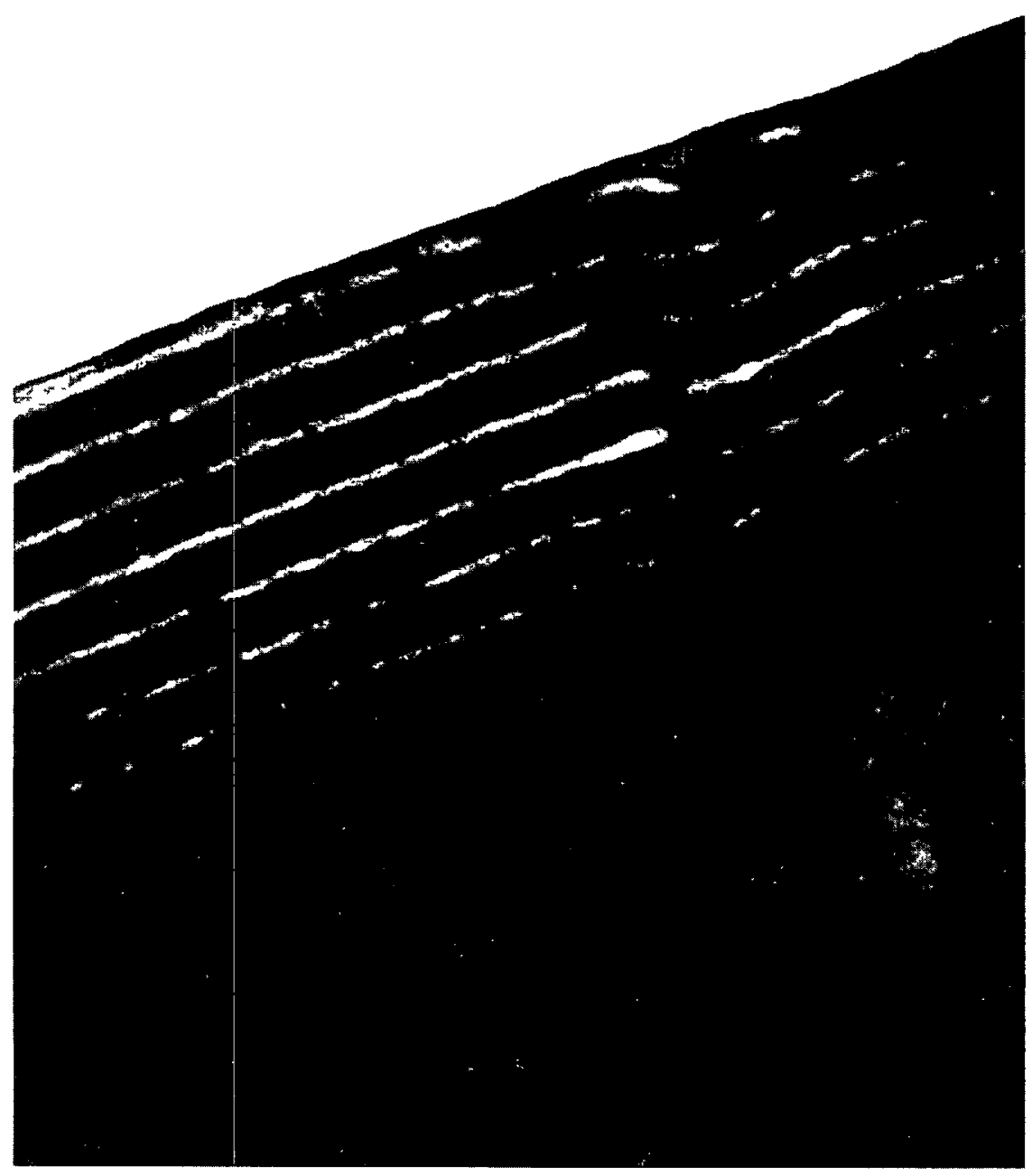

Fig. 9. - Cross-sectional TEM micrograph of a PS-PMMA film with a total thickness $H_{\text {tot }}$ such that $13 L / 2<H_{\text {tot }}<15 L / 2$. PS is black and PMMA white. The lower part of the picture (black) is the polymeric substrate.

this work but they are made of an odd instead of an even number of monolayers. A crosssectional slide of about $500 \AA$ thickness was made according to the preparation technique reported earlier $[19,20]$. Staining of the PS phase was achieved by treatment of the section with $\mathrm{RuO}_{4}$ vapor. Diffusion of the staining agent in the direction normal to the lamellae is probably responsible for the dissymmetry between the apparent thicknesses of PS and PMMA layers. However, the lamellar period $L=400 \AA$, is in good agreement with previous results on the same material [21] and the topology of the defects is expected to remain unaffected. The picture clearly demonstrates the presence of a dislocation inside the film. Moreover, it proves that such dislocations do not always lie against the free surface of the film. It is difficult to draw any other reasonable conclusions concerning the location of the defect or the bending of the trapped semi-infinite layer. Indeed, the substrate is rather rough and the films too thin to allow for more accurate comparisons with the model. 


\section{Conclusions.}

In this paper, we have presented a study of the domains which form in one incomplete layer of a lamellar copolymer film. The associated edge dislocations have been observed directly by transmission electron microscopy. The precise shape of the free surface above the edge of the domain results from a balance between the elastic distortion created by the dislocation and the surface tension at the copolymer-air interface. The role of surface tension is apparent when one compares the present results with the classical theory of Pershan [11], where surface tension effects were neglected: Pershan's prediction for the so-called healing length is much smaller than the observed one. The present work demonstrates that when surface tension is taken into account, the profile can be accurately predicted.

Our theoretical model is based on a continuum description of a freely suspended smectic film. It includes the effect of bending and compression as well as the surface tensions at the upper and lower film surfaces. We have given special attention to the case where the lower sample surface is rigid and flat and the upper surface is free. We find that the equilibrium position of the dislocation is at the free surface whenever the dimensionless surface tension $\Gamma_{\text {up }}=\gamma_{\text {up }} / \sqrt{K B}$ is less than unity. As $\Gamma_{\text {up }}$ is increased above unity the equilibrium dislocation position moves away from the surface, approaching the sample centre as $\Gamma_{\text {up }} \rightarrow \infty$. The experimental system PS/PBMA exhibits the rather large value $\Gamma_{\text {up }} \simeq 27$.

A direct calculation of the lamellar displacement field involves the numerical evaluation of a Fourier transform, in constrast to the infinite sum over image dislocations required in the representation of Lejcek and Oswald [12]. This may prove more convenient in many situations. We find that the conformation of the upper lamellar contour can be given in a simple closed form in the special case where $\Gamma_{\text {up }}=1$. For $\Gamma_{\text {up }} \gg 1$ the width of the free surface profile $\lambda$ varies as $\lambda \simeq\left(\kappa H_{\text {tot }} \Gamma_{\text {up }}\right)^{1 / 2}$ where $\kappa=\sqrt{K / B}$ is the smectic penetration length related to the mechanical properties of the lamellar material and $H_{\text {tot }}$ is the total sample thickness. This provides a simple explanation of the discrepancy between the experimental results and the classical theoretical estimate [11] of $\lambda$.

The experimental results obtained by atomic force microscopy are well described by this theoretical model even for the smallest film thicknesses, where it would appear at first sight that the discrete nature of the layers is an essential feature. Although for each film thickness the fit of the experimental data involves three parameters, we have some confidence in the results since the material parameters are actually independent of thickness. This allows a determination of the elastic constants of the smectic and an estimation of the internal interfacial tension between the two blocks of the copolymer. The results are in agreement with other estimates. The depth of the dislocation below the free surface is also obtained and could be compared to more systematic observations by electron microscopy.

Future developments of this work could involve a more thorough study of the interaction between dislocations and of the kinetics of relaxation to its equilibrium position upon annealing.

\section{Appendix A.}

\section{Equilibrium dislocation position: sample at a flat substrate.}

For the case when $\Gamma_{\text {up }}=1+\delta$ with $\delta \ll 1$ we calculate the equilibrium dislocation depth $H_{\text {up }}$ from (11) by assuming that $H_{\text {up }}=\varepsilon H_{\text {tot }}$ with $\varepsilon \ll 1$. We then expand (11) in powers of $\delta$ and $\varepsilon$, 
keeping only the leading order terms. Minimization of $F^{\text {tot }}$ with respect to $H_{\text {up }}$ then leads to

$$
H_{\text {up }}=H_{\text {tot }}(\delta / 2)^{2 / 3}
$$

where we recall that $\delta=\Gamma_{\text {up }}-1$ with $\delta \ll 1$.

\section{Appendix B.}

\section{Calculation of $\lambda$ for $\Gamma_{\text {up }} \gg 1$.}

For the case when the surface tension at the free surface is very large $\Gamma_{\text {up }}=1 / \delta$ with $\delta \ll 1$ we have $G_{\text {up }}=-(1+2 \delta)$ to leading order in $\delta$. We assume that $H_{\text {up }} \approx H_{\text {tot }} / 2$ and expand (14) in powers of $\delta$, keeping only the leading order terms. In order to calculate $\lambda$ one needs to estimate $\left.\frac{\partial u}{\partial x}\right|_{x=0}$, which involves an integral over all $q$-space. However the contribution from the range $q=[0, \tilde{q}]$, with $\tilde{q}^{2} \simeq \delta / \kappa H_{\text {tot }}$, dominates the integral. An estimate of this then gives equation (18), to within a numerical prefactor of order unity.

\section{Acknowledgments.}

The authors are grateful to J. Prost (ESPCI Paris) and P. Oswald (E.N.S. Lyon) for useful discussions.

\section{References}

[1] Bates F. S., Fredrickson G. H., Annu. Rev. Phys. Chem. 41 (1990) 525.

[2] Coulon G., Collin B., Ausseré D., Chatenay D., Russell T. P., J. Phys. France 51 (1990) 2801.

[3] Collin B., Chatenay D., Coulon G., Ausseré D., Gallot Y., Macromolecules 25 (1992) 1621.

[4] Anastasiadis S., Russell T., Satija S., Majkrzaka C., Phys. Rev. Lett. 62 (1989) 1852.

[5] Anastasiadis S., Russell T., Satija S., Majkrzaka C., J. Chem. Phys. 92 (1990) 5677.

[6] Coulon G., Russell T., Deline V., Green P., Macromolecules 22 (1989) 2581.

[7] Maaloum M., Ausserré D., Chatenay D., Coulon G., Gallot Y., Phys. Rev. Lett. 68 (1992) 1575.

[8] de Gennes P. G., The Physics of Liquid Crystals (Clarendon, Oxford, 1974).

[9] de Gennes P. G., J. Phys. Colloq. France 30 (1969) C4-65.

[10] Kleman M., Points, Lines and Surfaces (Edition de Physique, Paris, 1978).

[11] Pershan P. S., J. Appl. Phys. 45 (1974) 1590.

[12] Lejcek L., Oswald P., J. Phys. II France 1 (1991) 931.

[13] Turner M. S., Joanny J-F., Macromolecules 25 (1992) 6681.

[14] Semenov A. N., Sov. Phys.-JETP (Eng. Transl.) 61 (1985) 733.

[15] Abramowitz M., Stegun I., Handbook of mathematical functions (Dover, N.Y., 1972), p. 1028.

[16] Maaloum M., PhD thesis, University of Paris VI (1993).

[17] Gallot Y., Private communication.

[18] Wu S., J. Phys. Chem. 74 (1970) 632.

[19] Kunz M., Shull K., Polymer Commun. 34 (1993) 2427.

[20] Kunz M.,Russell T., Mayes A., Nature, submitted for publication.

[21] Ausseré D., Chatenay D., Coulon G., Collin B., J. Phys. France 51 (1990) 2801. 